\title{
Changes in Bending Stiffness and Lumbar Spine Range of Movement following Mobilization and Manipulation
}

\author{
Nikolaos Stamos-Papastamos, MSc, Nicola J. Petty, DPT, and Jonathan M. Williams, MManipTher
}

\begin{abstract}
Objective: The purpose of this study was to investigate the effects of lumbar rotational manipulation and lumbar central posteroanterior mobilization on lumbar bending stiffness and flexion and extension range of motion (ROM). Methods: A samesubject, repeated-measures, crossover design was used using 32 asymptomatic subjects (16 female and 16 male; mean [SD] age, 25.5 [4.5] years; weight, 65.7 [11.8] kg; and height, 1.70 [0.08] m). Each subject received mobilization or manipulation on 2 different occasions. Bending stiffness was calculated using a 3-point bending model using an electromagnetic tracking device and a force platform; lumbar flexion and extension ROM was measured using an electromagnetic tracking device. All variables were measured pre- and postintervention. Their effect was compared using paired $t$ tests.

Results: Manipulation and mobilization did not significantly alter either bending stiffness or lumbar flexion and

extension $\mathrm{BOM}$ (mobilization: $P=.175, P=613$, and $P=535$; manipulation: $P=.973, P=.323$. and $P=5439)$.

Pearson $r$ for stiffness-extension $=0.014, P=.941$ ).

Conclusions: Manipulation and mobilization had no significant effect on bending stiffness or flexion and extension ROM for this group of subjects. Some individual variations in effect were observed.
\end{abstract}

ow back pain (LBP) is a common health problem with important socioeconomic impact on its sufferers The costs incurred by health care systems,

including direct medical care expenses, as well as indirect costs resulting from lost time, reduced productivity, and disability allowance, are vast. ${ }^{2,3}$ Recent National Institute for Health and Clinical Excellence clinical guidelines ${ }^{4}$ have recommended that manual therapy (MT) may be beneficial in the treatment of patients with LBP. However, MT is a generic term that includes a wide range of interventions. The most commonly used forms of MT aimed at the lumbar spine include mobilization and manipulation. Mobilization is defined here as the use of repetitive, non-thrust forces applied to the spine; and manipulation is defined as a high-velocity

Page 1 of 8 small-amplitude thrust. ${ }^{5}$ Central posteroanterior $(\mathrm{PA})$ mobi- lization and rotation manipulation are commonly used treatment techniques by manual therapists. ${ }^{6-10}$ The underly- ing mechanism attributable to the clinical success of MT is not yet established, and many theories have been suggested ${ }^{11}$; however, because of poor clarity with definitions, it is unclear as to whether manipulation and mobilization have similar underlying mechanisms. A common biomechanical theory is that MT may alter spinal stiffness. ${ }^{12}$

Changes in bending stiffness properties of the lumbar spine in response to PA mobilization have been investigated previously, ${ }^{12-15}$ but have shown conflicting results. Bending stiffness is a term taken from materials testing that can be applied to the spine. The spine is modeled as a beam fixed at 2 points: the pelvis and ribcage. A load is applied to a third point on the spine, and the resulting deformation is measured. Therefore, bending stiffness as applied in this study is the relationship between load and deformation modeling the spine during 3-point bending. ${ }^{13}$ Allison et $\mathrm{al}^{14}$ following 2 minutes of PA mobilization found no effect on spinal stiffness, a finding mirrored by Shirley et $\mathrm{al}^{15}$ using 
only preconditioning mobilization. Furthermore, Goodsell et $\mathrm{al}^{16}$ using 3 minutes of PA mobilization found no effect on stiffness. Conversely, Lee et $\mathrm{al}^{12}$ showed that spinal stiffness was reduced after 3 minutes of PA mobilization.

These differences may be explained by the diversity in methodology. Spinal bending stiffness may be calculated from the relationship between force and displacement. ${ }^{17}$ Custom-made devices using a load cell and linear potentiometer to measure PA load and resultant linear displacement were used in some of the studies. ${ }^{14-}$

16 However, it has been argued that because the geometry of the lumbar spine is curved and undergoes further deformation of the curve in response to PA loading, it is more appropriate to model bending stiffness through the measurement of curvature change. $^{13,17,18}$ This method of measurement has been shown to be highly reliable with correlation coefficients between 0.97 and $0.99,{ }^{17}$ and the differences in these modeling methods may explain the inconsistencies reported in the literature.

Previous studies ${ }^{19-21}$ provide some evidence that increased PA stiffness could be linked to LBP. Latimer et $\mathrm{al}^{19}$ were able to show that LBP sufferers had elevated stiffness levels that decreased as the individual's pain level reduced. However, the authors did not statistically correlate LBP and PA stiffness. Similarly, Shirley ${ }^{20}$ found that PA stiffness decreased as LBP subsided in 15 LBP sufferers. The author found that subjects with higher pain had higher PA stiffness values, but there was no significant correlation between pain and stiffness. More recently, Ferreira et $a{ }^{21}$ found that PA stiffness can be reduced with mobilization or manipulation, but there was no significant correlation between change in stiffness and change in pain. Furthermore, the greatest treatment benefit was observed in those with the stiffest spines. It was also reported that a high correlation was evident for stiffness, range of motion (ROM), and pain in LBP sufferers as seen by Lee et al, ${ }^{12}$ suggesting an interaction between the 3 variables in LBP sufferers.

The effect of mobilization on ROM has also shown inconsistency. McCollam and Benson ${ }^{22}$ found that 9 minutes of PA mobilization increased participants' extension ROM by $7.1 \%$, but had no effect on flexion ROM. Lee et $\mathrm{al}^{12}$ found a $4.3 \%$ increase in flexion and a $27.2 \%$ increase in extension following 3 minutes of PA mobilization. In contrast, Petty ${ }^{23}$ found no change in ROM following 2 minutes of PA mobilization. These discrepancies may be explained by differences in sex, as an all-female sample was used by Petty, ${ }^{23}$ which may characteristically have less initial stiffness. ${ }^{24}$ Furthermore, variations in the mobiliza- tion protocol may also explain some of the differences.

The effect of manipulation on stiffness has not been well investigated in the literature. It has been shown that thoracic manipulation had no effect on thoracic spine bending stiffness $^{25,26}$; however, to the authors' knowledge, no studies have investigated the effect of lumbar manipulation on bending stiffness.
Few studies examined the effect of lumbar manipulation on ROM. Lehman and McGilll ${ }^{27}$ found that manipulation resulted in significant gains in ROM, but only in a singlesubject design. In a larger study, the same authors did not find consistent effects of manipulation on ROM. ${ }^{28}$ Evidence from other spinal regions suggests that manipulation could increase ROM; however, this cannot be directly extrapolated to the lumbar spine because of biomechanical differences of the anatomical regions. ${ }^{29-33}$

To gain knowledge regarding the mechanism of effect of $M T$, it is necessary to investigate the relationship between stiffness and ROM. Lee et $\mathrm{al}^{12}$ showed that the ROM correlated significantly to the measured stiffness (0.77). Although it is acknowledged that correlation cannot imply causation, this relationship aids understanding of the response to MT.

It remains unclear whether different MT interventions are interchangeable because of a consistent response and underlying mechanism of action. Recent evidence suggests that the clinical response to manipulation may be marginally different to that of PA mobilization in LBP patients. ${ }^{34}$ Therefore, it needs to be determined if mobilization and manipulation have different effects on stiffness and ROM and if any changes correlate to changes in ROM. Past research ${ }^{12}$ identified significant changes on bending stiffness after PA mobilization on subjects without LBP. In addition, earlier manipulation studies did not correlate changes in ROM and changes in bending stiffness on either LBP patients $^{27,28}$ or asymptomatic subjects..$^{25,26}$ It was hypothesized that a direct comparison of the 2 techniques on the same asymptomatic subjects could possibly clarify the interaction of bending stiffness and ROM, without pain being present. Moreover, any changes on stiffness and ROM after the application of either technique on asymptomatic subjects could not be due to pain relief.

The aims of the present study were to:

1. Investigate and compare the immediate effects of lumbar PA mobilization and lumbar manipulation on spinal bending stiffness and lumbar ROM.

2. Assess the relationship between changes of bending stiffness and lumbar ROM.

\section{Methods}

Subjects

Thirty-two asymptomatic subjects, 16 female and 16 male, were recruited by poster advertisement from the School of Health Professions, University of Brighton. All subjects were undergraduate students in the University of Brighton. Ethical approval for the study was granted by the School Research Ethics and Governance Panel, and all subjects provided written informed consent. Subjects were excluded if they had any history of back pain, spinal surgery, tumors, or any other disorders that may be aggravated by the testing procedures. ${ }^{35,36}$ To achieve a 


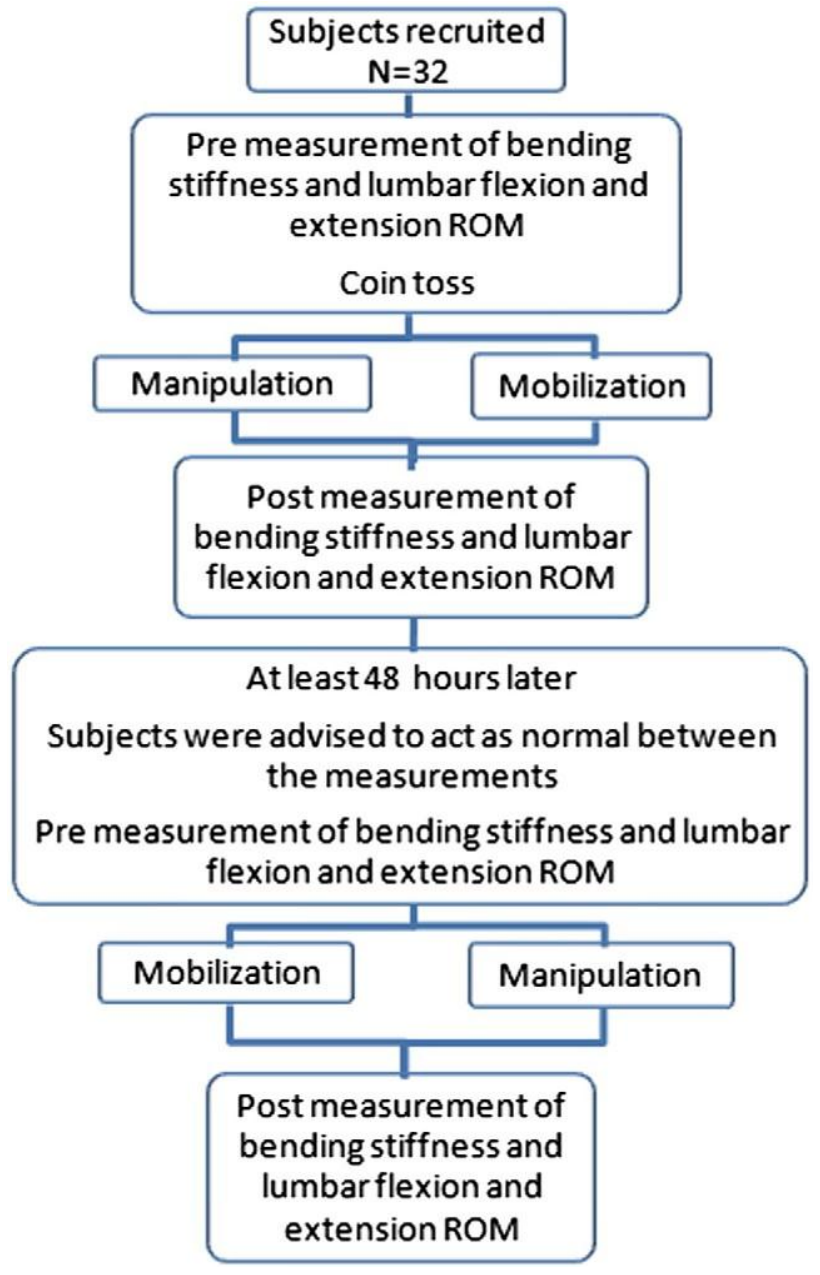

Fig 1. Design of the study.

power of 0.8 with an a level set at 0.05 , a sample size calculation based on the literature revealed that a sample of 31 was necessary. ${ }^{12}$

\section{Study Design}

A same-subject, repeated-measures, crossover design was used. Dependent variables were bending stiffness and lumbar ROM. These measures were taken before and after the mobilization or manipulation intervention, as shown in Figure 1. All measurements and interventions were performed by the researcher (NSP) who had specialist training in MT and more than 5 years of clinical experience.

\section{Instrumentation}

A custom-made wooden and padded treatment plinth securely screwed on a nonconductive force platform (Advanced Mechanical Technology Inc, Watertown, MA) was used to quantify the magnitude of PA force applied to the spine. A similar setup has been used in previous studies. ${ }^{12,17,37}$ An electromagnetic tracking system (Fastrak; Polhemus Navigation, Colchester, VT), recording at $100 \mathrm{~Hz}$, was used to measure spinal angular displacement during force application for the calculation of bending stiffness ${ }^{37-}$ and to measure lumbar flexion and extension ROM. This instrumentation has been shown to be highly reliable to

$\pm 0.2^{\circ 38}$ as well as highly accurate when compared with radiographs ${ }^{39,40}$ for the measurement of lumbar ROM.

\section{Interventions}

Subjects lay prone on the plinth that was mounted on the force platform, and the location of spinous processes $\mathrm{L} 1, \mathrm{~L} 4$, and $\mathrm{S} 1$ was marked by the researcher (NSP). Subject position was standardized by marking the plinth at the toes and acromioclavicular joints. Electromagnetic sensors were adhered to the skin overlying the spinous processes of S1 and L1 using double-sided tape and reinforced by additional tape. Before any data collection, 3 central PA pressures were applied to the spinous process of $L 4$ with the subject in prone position. This was done to familiarize the subjects with the PA pressures, to ensure that they could tolerate the forces applied and to precondition the spine preventing any artifact readings. ${ }^{19}$ Subsequently, 5 central PA pressures were applied to L4 to collect measurements of force and angular displacement for the calculation of bending stiffness. Lumbar flexion and extension ROM was measured using a standardized protocol, with total ROM measured relative to a standing reference position. Markers were taped to the floor, $20 \mathrm{~cm}$ apart, to standardize the position of the feet; and subjects were asked to look directly ahead, standing erect with arms by the sides and knees straight. This position was adopted as their zero reference position, and all ROM measurements were calculated with respect to this. ${ }^{37}$ The subjects were given standard instructions to bend forward and backward as far as possible, keeping knees fully extended. The data collected represented the difference in angular displacement between the 2 sensors, thereby overcoming the problem of pelvis contribution to the movement.

Subjects then received 1 of the 2 MT interventions, randomized by coin toss. Following a minimum of 48 hours, the subjects returned and received the alternate intervention. No attempt was made to restrict subject activity between sessions. The manipulation intervention involved a rotational manipulative thrust technique on both sides of the L4/5 segmental level as described by Gibbons and Tehan. ${ }^{41}$ The mobilization intervention consisted of 3 sets of 1 minute of PA mobilization into the researcher's perceived resistance, aiming at grade IV+ as described by Maitland. ${ }^{5}$ The researcher throughout the application of the technique used a pisiform contact, and all subjects were lying prone. A mobilization frequency of $2 \mathrm{~Hz}$ was maintained by a metronome. Following each intervention, PA bending stiffness and lumbar ROM were remeasured. 
Table 1. The mean (SD) stiffness and ROM measures pre- and postmobilization and manipulation with $t$ test $P$ values to measure significance

\begin{tabular}{llll}
\hline & \multicolumn{3}{c}{ Mobilization } \\
\cline { 2 - 4 } & Pre & Post & Pvalue \\
\hline Stiffness $\left(\mathrm{N} /{ }^{\circ}\right)$ & $20.78(17.24)$ & $25.05(22.57)$ & .175 \\
Flexion $\left({ }^{\circ}\right)$ & $54.11(11.13)$ & $54.81(11.36)$ & .613 \\
Extension $\left({ }^{\circ}\right)$ & $22.8(10.0)$ & $23.66(11.39)$ & .535 \\
& \multicolumn{3}{c}{ Manipulation } \\
\cline { 2 - 4 } & Pre & Post \\
\hline Stiffness $\left(\mathrm{N} /{ }^{\circ}\right)$ & $23.14(19.66)$ & $23.04(18.52)$ & .973 \\
Hlexion $\left({ }^{\circ}\right)$ & $54.22(12.76)$ & 56.07 & .323 \\
Extension $\left({ }^{\circ}\right)$ & $27.02(14.42)$ & 28.56 & .439 \\
\hline
\end{tabular}

All measurements were performed by an independent researcher who volunteered to operate the software after a small training period, and all data processing was calculated by automated computer processes.

\section{Data Analysis}

Posteroanterior bending stiffness was calculated from 5 PA loading cycles applied with the subject lying prone on the plinth mounted on the force platform. All loading cycles for stiffness calculations were completed by the same experienced manual therapist who attempted to provide identical loading cycles. Angular displacement data, derived from the electromagnetic tracking device, was divided by PA force data from the force plate for the second, third, and fourth PA loading cycle. All data were processed and calculated using Windows Excel (Microsoft, Redmond, Wash, 2007). Lumbar flexion and extension ROM measurements were calculated using the mean of 6 movement trials. Paired $t$ tests were performed (SPSS, 16.0, Chicago, IL) to examine the effects of intervention on bending stiffness and lumbar ROM. A 2-way analysis of variance determined the effect of the technique order. Pearson correlation analysis was used to determine the relationship between stiffness and lumbar ROM.

\section{Results}

All data were normally distributed as found by the Kolmogorov-Smirnov test $(P=.115-.998)$. Subjects' mean (SD) age was 25.5 (4.5) years, and body mass index was

$22.05(3.78) \mathrm{kg} / \mathrm{m}^{2}$. The mean (SD) magnitude of force measured during PA mobilization intervention was 154.3 (12.4) N. The analysis of variance showed that technique order had no effect on the results $(P=.804)$. The effects of mobilization and manipulation on the variables of stiffness and lumbar ROM can be seen in Table 1. Manipulation and mobilization had no effect on bending stiffness or lumbar flexion and extension ROM of the group as a whole.
There was no significant correlation between changes on bending stiffness and ROM (Pearson $r$ for stiffness-

flexion $=-0.102, P=.586$; Pearson $r$ for stiffness-extension $=$ $0.014, P=.941)$.

\section{Discussion}

The results of the study show that PA mobilization and spinal manipulation did not significantly alter bending stiffness or lumbar flexion and extension ROM. The study's findings offer the first insight into the effects of manipulation on bending stiffness in asymptomatic subjects. They are in agreement with studies investigating bending stiffness in other anatomical regions. ${ }^{25,26}$ It appears that manipulation is unable to influence spinal bending stiffness in asymptomatic subjects.

The results of this study that mobilization had no effect on bending stiffness are in agreement with those of Allison et $\mathrm{al}^{14}$ and Goodsell et $\mathrm{al}^{16}$; on the other hand, they are in contrast to those of Lee et al. ${ }^{12}$ This may be due to differences in the stiffness measurement methodology, making direct comparison of stiffness values not possible, or due to differences in the subjects' characteristics. The finding that lumbar manipulation had no effect on range of movement is in agreement with previous manipulation studies, ${ }^{25,26}$ but is in contrast to Lehman and McGill. ${ }^{27,28}$ The reason for this difference may lie in the sample characteristics and the fact that all their subjects were experiencing LBP. However, a trend was noted in that the participants who increased their ROM were those who initially had higher Oswestry disability scores, suggesting that initial characteristics may be important in predicting the effect. ${ }^{28}$

Further observation of the data and analysis of the present study revealed some possible trends in response to treatment. Identification of individual characteristics to detect those likely to respond was carried out using the available data from the study. It appeared that baseline ROM influenced the likelihood of the subject to respond to the interventions. The authors hypothesized that baseline ROM could determine the outcome of interven- tion, and this was investigated. Seventy-three percent of those whose initial lumbar extension was less than $20^{\circ}$ showed an increase in extension after PA mobilization (Fig 2). Seventy-four percent of the subjects with an initial range of less than $56^{\circ}$ of lumbar flexion increased their ROM by $12 \%$ following mobilization. Fifty-eight percent of subjects with more than $56^{\circ}$ flexion ROM showed an $11 \%$ decrease in ROM following mobilization (Fig 3). Similar trends were demonstrated with manipulation. In those subjects with less than $27^{\circ}$ of initial extension, $62.5 \%$ increased their extension ROM with manipulation, whereas in those with greater than $27^{\circ}$ extension, 58\% decreased extension ROM following manipulation (Fig 4). Almost half of the participants $(56.3 \%)$ who had initial 


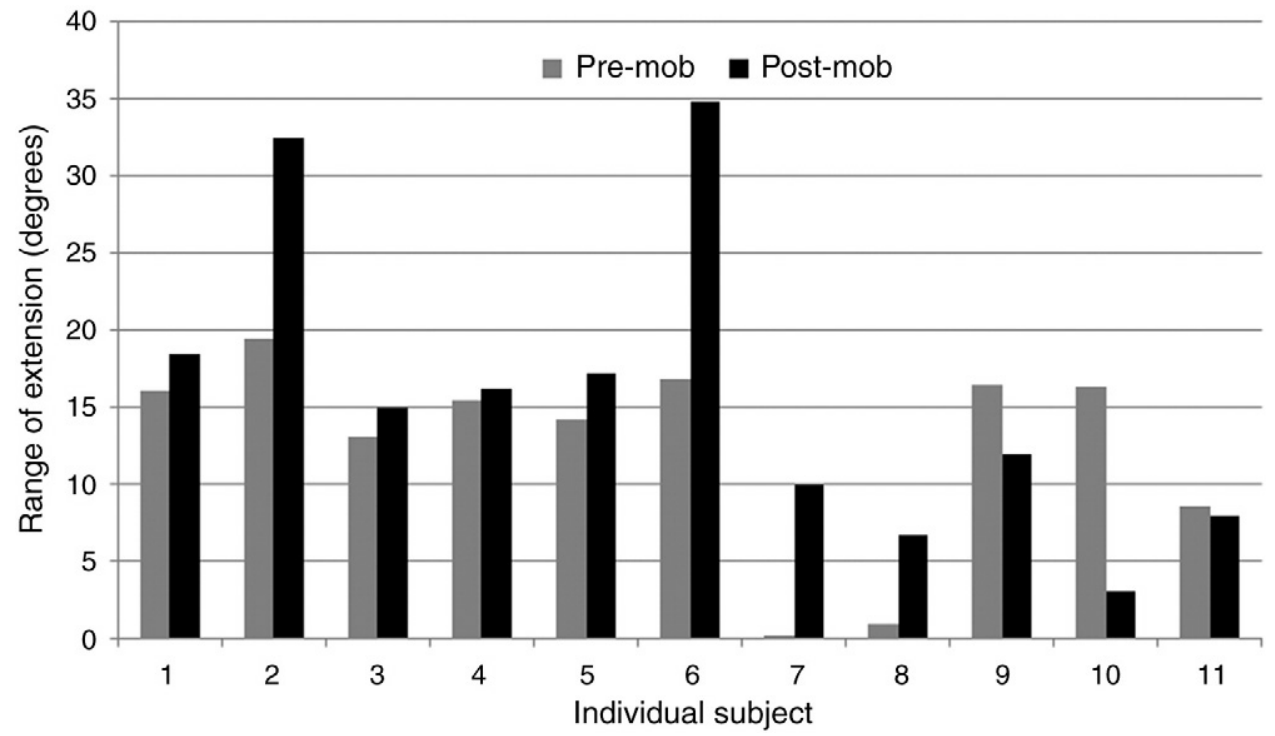

Fig 2. Data series showing effect of PA mobilization on subjects displaying an initial extension ROM of less than $20^{\circ}$.

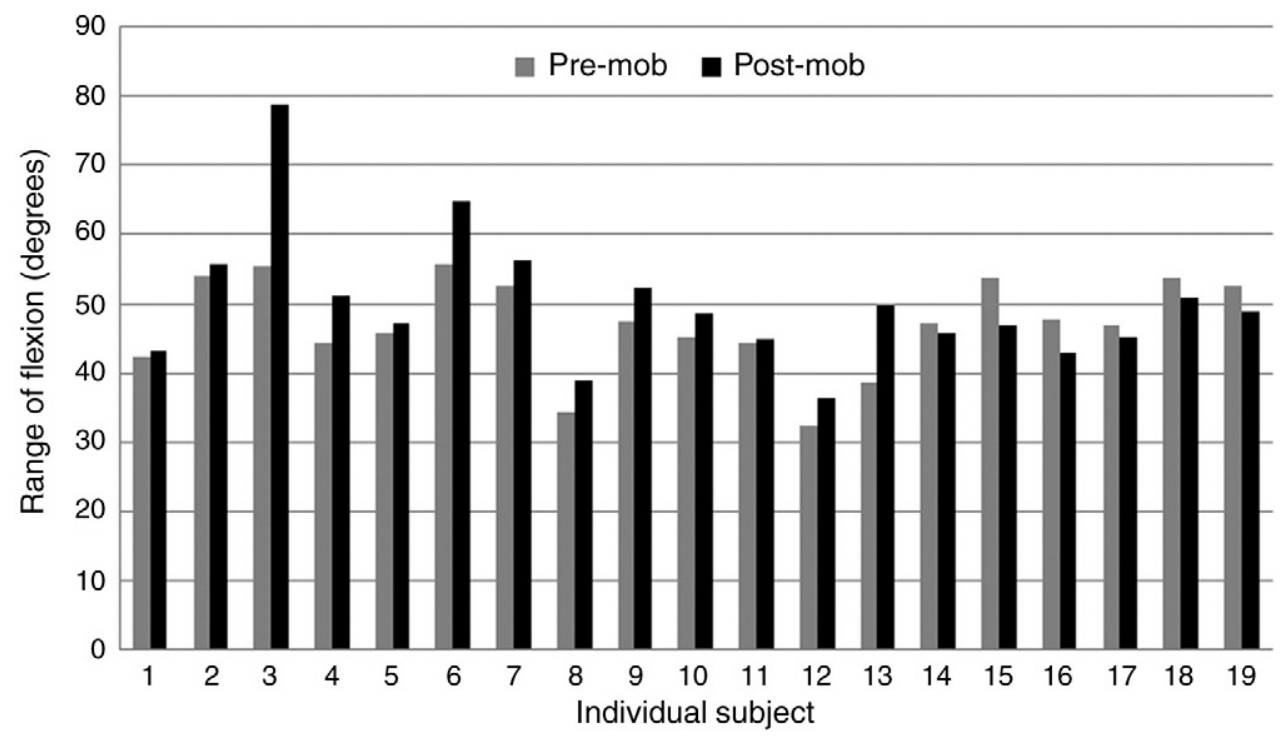

Fig 3. Data series showing effect of PA mobilization on subjects displaying an initial flexion ROM of less than $56.3^{\circ}$.

flexion $\mathrm{ROM}$ less than $54^{\circ}$ increased flexion range with manipulation, whereas $58 \%$ of those who had more than $53.6^{\circ}$ of initial flexion ROM decreased flexion ROM following manipulation (Fig 5).

In addition, some links were observed between changes in stiffness and changes in lumbar ROM. Fifty percent of subjects in whom manipulation reduced bending stiffness showed large gains in extension ROM (67\%). Fiftyseven percent of subjects in whom mobilization increased bending stiffness showed a decrease in extension ROM $(23 \%)$.

These findings agree with the trends identified in Lehman and McGills'28 study, which suggests that those with less initial ROM are more likely to respond to manipulation.
The lack of effect of mobilization on ROM is in agreement with Petty's ${ }^{23}$ study but in contrast with the findings of Lee et al. ${ }^{12}$ Initial ROM may help explain the discrepancies observed. Subjects in the current study with initial extension ROM greater than $20^{\circ}$ were less likely to increase ROM following mobilization. This is in agreement with Petty $^{23}$ whose sample demonstrated premobilization extension ROM greater than $35^{\circ}$ and had no response to mobilization. Conversely, other studies ${ }^{12,22}$ did show an improvement in lumbar flexion and extension following mobilization. Lee et $\mathrm{al}^{12}$ found significant gains in flexion following a similar mobilization protocol to that used in the present study. Importantly, those participants in the study by Lee et $\mathrm{al}^{12}$ 


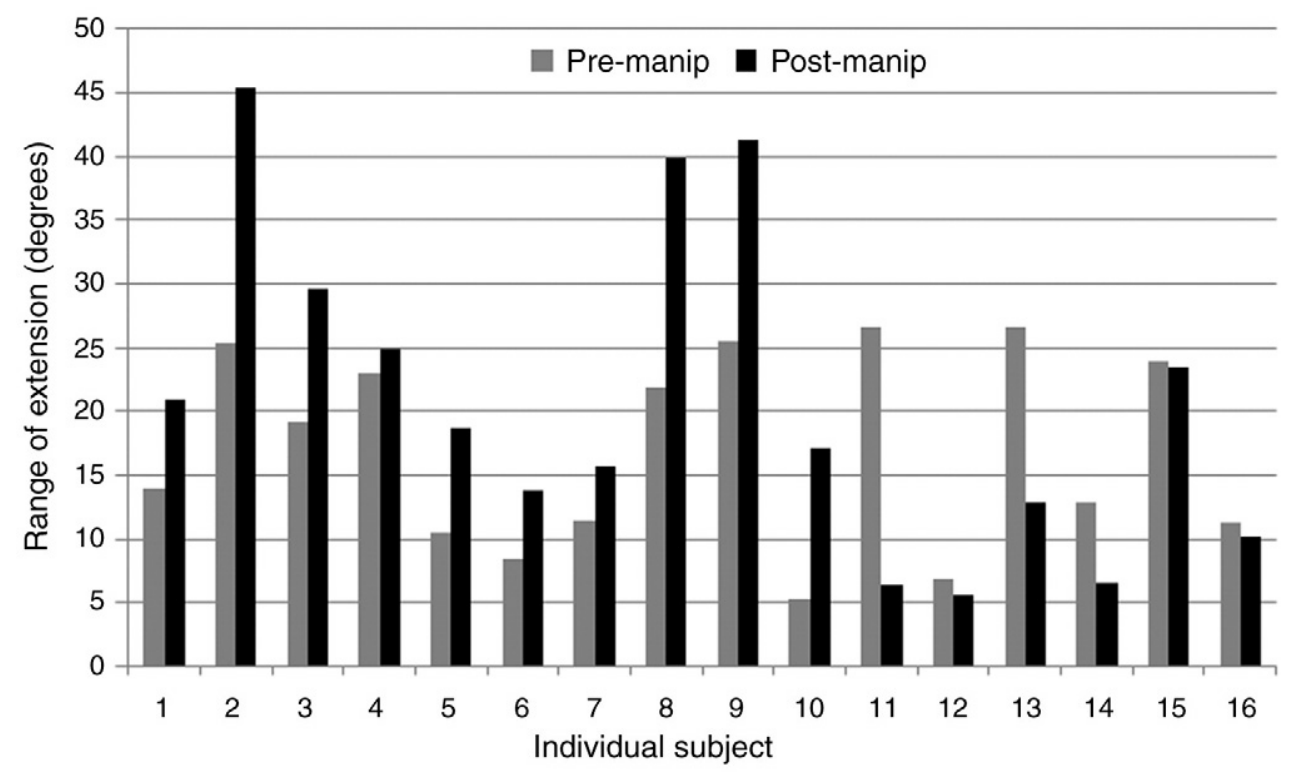

Fig 4. Data series showing effect of manipulation on subjects displaying an initial extension ROM of less than $27^{\circ}$.

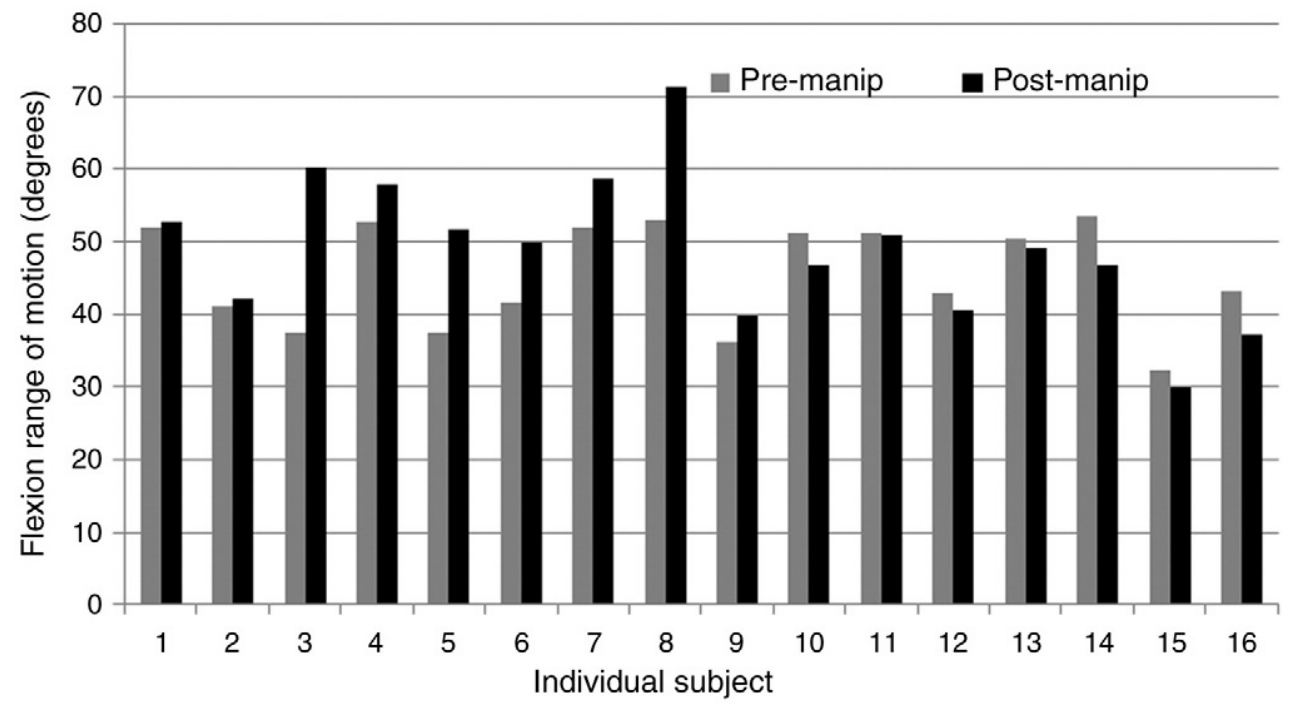

Fig 5. Data series showing effect of manipulation on subjects displaying an initial flexion ROM of less than 54․

demonstrated small initial ranges (less than the cutoff identified in this study) with a mean premobilization range of $58.3^{\circ}$ of flexion and $18^{\circ}$ of extension, which may explain the difference between this and the present study. McCollam and Benson ${ }^{22}$ also found significant improvements in lumbar extension after PA pressures with pre extension range of $28^{\circ}$. The treatment protocol was given for a longer period (9 minutes) and multiple segments were mobilized, which may also help explain the conflicting results.

\section{Limitations}

The present study was conducted using young asymptomatic subjects and therefore cannot be extrapolated to back pain sufferers or to the general population. Asymptomatic subjects were selected to avoid the confusion of cause and effect. The study's aim was to investigate the effect of mobilization and manipulation on stiffness and ROM, not to study the effects of pain relief (common following mobilization and manipulation on painful sub- jects) on stiffness and ROM. This enables more specific conclusions to be drawn about the intervention on stiffness and ROM directly, not secondarily due to pain relief. This study investigated the immediate effect of MT, preventing the generalization to more long-term effects. The measure- ment of stiffness requires the application of a force delivered by the investigator and therefore was not strictly controlled. 
This may have affected the stiffness measurements, as the stiffness response is not linear; however, it has been shown that experienced manual therapists are remarkably consis- tent in the application of PA force. ${ }^{42,43}$ It is possible that the preconditioning pressures might have affected any initial stiff segments before the calculation of stiffness and contributed to the results of the study. However, precondi- tioning has been shown to be important because of the spine's viscoelastic properties; and the stiffness values collected during these preconditioning loads have been reported to be erroneous. ${ }^{19}$ No attempts were made to restrict normal activities between interventions; thus, it is possible that these activities may have affected the results of the study. This could have contributed to the discrepancies between the cutoff points of ROM in mobilization and manipulation. The authors acknowledge that this might not be the case for other samples. There is a risk of operator bias because the independent researcher, who participated in the data collection, was not blinded to the measurements. However, this risk was minimized by the use of automated computer algorithms for data processing.

\section{Conclusion}

The results of the present study show that PA mobilization and rotational spinal manipulation as applied to young asymptomatic subjects did not significantly alter PA bending stiffness or lumbar ROM. No clear relationship between stiffness and ROM could be identified. Further analysis suggests possible differences in response to mobilization and manipulation depending on the magnitude of motion before the procedure. Further work investigating the effects of manipulation and mobilization should be reserved for subjects whose baseline characteristics include limited ROM.

\section{References}

1. Du Bois M, Szpalski M, Donceel P. Patients at risk for longterm sick leave because of low back pain. Spine $\mathrm{J}$ 2008;9: 350-9.

2. Atlas $\mathrm{S}$, Deyo R. Evaluating and managing acute low back pain in the primary care setting. $J$ Gen Intern Med 2001;16: 120-31.

3. Dagenais S, Caro J, Haldeman S. A systematic review of low back pain cost of illness studies in the United States and internationally. Spine J 2008;8:8-20.

4. National Institute for Health and Clinical Excellence. Low back pain. Early management of persistent non-specific low back pain. London: NICE; 2009. p. 25. Available from: http:// www.nice.org.uk/CG88.

5. Maitland GD. Vertebral manipulation. 7th ed. London: Butterworths; 2007.

6. Lee M. Mechanics of spinal joint manipulation in the thoracic and lumbar spine: a theoretical study of posteroanterior force techniques. Clin Biomech 1989;4:249-51.

7. Shirley D, Lee M. A preliminary investigation of the relationship between lumbar posteroanterior mobility and low back pain. J Man Manip Ther 1993;1:22-5.

8. Jull G. Cervicogenic headache. In: Grant R, editor. Physical therapy of the cervical and thoracic spines. 3rd ed. New York: Churchill Livingstone; 2002. p. 239-72.

9. Magarey M, Rebbeck T, Coughlan B, Grimmer K, Rivett D, Refshauge K. Pre-manipulative testing of the cervical spine review, revision and new clinical guidelines. Man Ther 2004; 9:95-108.

10. Snodgrass S, Rivett D, Robertson V. Manual forces applied during posterior-to-anterior spinal mobilization: a review of the evidence. J Man Manip Ther 2006;29:31629.

11. Zusman M. Spinal manipulative therapy: review of some of proposed mechanisms, and a new hypothesis. Aust J Physiother 1986;32:89-99.

12. Lee R, Tsung B, Tong $P$, Evans J. Posteroanterior mobilization reduces the bending stiffness and the pain response in the lumbar spine. Proceedings of the 2 nd International Conference on Movement Dysfunction, Edinburgh;2005. p. 39.

13. Lee R, Evans J. An in vivo study of the intervertebral movements produced by posteroanterior mobilization. Clin Biomech 1997:12:400-8.

14. Allison G, Edmonston S, Kiviniemi K, Lanigan $\mathrm{H}$, Simonsen $\mathrm{AV}$, Walcher $\mathrm{S}$. Influence of standardized mobilization on the posteroanterior stiffness of the lumbar spine in asymptomatic subjects. Physiother Res Inter 2001;6:145-56.

15. Shirley D, Ellis $E$, Lee $M$. The response of posteroanterior lumbar stiffness to repeated loading. Man Ther2002;7:19-25

16. Goodsell M, Lee M, Latimer J. Short-term effects of lumbar posteroanterior mobilization in individuals with low-back pain. J Man Manip Ther 2000;23:332-42.

17. Lee $R$, Tsung $B$, Tong $P$, Evans J. Bending stiffness of the lumbar spine subjected to posteroanterior manipulative force. J Rehabil Res Dev 2005;42:167-74.

18. Lee R, Evans J. Load-displacement-time characteristics of the spine under posteroanterior mobilization. Aus $\mathrm{J}$ Physiother 1992;38:115-24.

19. Latimer J, Lee M, Adams R, Moran CM. An investigation of the relationship between low back pain and lumbar posteroanterior stiffness. J Manipulative Physiol Ther 1996;19:587-91.

20. Shirley D. Muscle activity and lumbar PA stiffness. [PhD thesis]. Sydney: University of Sydney; 2002.

21. Ferreira M, Ferreira P, Latimer J, Herbert R, Maher C, Refshauge K. Relationship between spinal stiffness and 
outcome in patients with chronic low back pain. Man Ther 2009;14:61-7.

22. McCollam R, Benson C. Effects of posteroanterior mobilization on lumbar extension and flexion. J Man Manip Ther 1993;1:134-41.

23. Petty $\mathrm{N}$. The effect of posteroanterior mobilization on sagittal mobility of the lumbar spine. Man Ther 1995;1:25-9.

24. Owens E, DeVocht J, Gudavalli R, Wilder D, Meeker W. Comparison of posteroanterior spinal stiffness measures to clinical and demographic findings at baseline in patients enrolled in a clinical study of spinal manipulation for low back pain. J Manipulative Physiol Ther 2007;30:493-500.

25. Latimer J, Lee M, Maher C. Effects of manipulations on the thoracic spine. Proceedings of the 11 th International Congress of the World Confederation for Physical Therapy, London; 1991. p. 371-3.

26. Lee M, Latimer J, Maher C. Manipulation: investigation of a proposed mechanism. Clin Biomech 1993;8:302-6.

27. Lehman G, McGill S. The influence of a chiropractic manipulation on lumbar kinematics and electromyography during simple and complex tasks: a case study. J Manipulative Physiol Ther 1999;22:576-81.

28. Lehman G, McGill S. Spinal manipulation causes variable spine kinematic and trunk muscle electromyographic responses. Clin Biomech 2001;16:293-9.

29. Nansel D, Peneff A, Cremata E, Carlson J. Time course considerations for the effects of unilateral lower cervical adjustments with respect to the amelioration of cervical lateral flexion passive end-range asymmetry. J Manipulative Physiol Ther 1990;13:294-7.

30. Cassidy JD, Lopes AA, Yong-Hink K. The immediate effect of manipulation versus mobilization on pain and range of motion in the cervical spine: a randomized controlled trial. J Manipulative Physiol Ther 1992;15:570-5.

31. Schalkwyk RV, Parkin-Smith GF. A clinical trial investigating the possible effect of the supine cervical rotatory manipulation and the supine lateral break manipulation in the treatment of mechanical neck pain: a pilot study. J Manipulative Physiol Ther 2000;23:324-31.

32. Martinez-Segura R, Fernandez-de-las-Penas C, Ruiz-Saez $M$, Lopez-Jimenez C, Rodriguez-Blanco C. Immediate effects on neck pain and active range of motion after a single cervical high-velocity low-amplitude manipulation in subjects presenting with mechanical neck pain: a randomized controlled trial. J Manipulative Physiol Ther 2006;29:511-7.

33. Fernandez-de-las-Penas $C$, Palomeque-del-Cerro $L$ Rodriguez- Blanco C, Gomez-Conesa A, Miangolarra-Page $J$. Changes in neck pain and active range of motion after a single thoracic spine manipulation in subjects presenting with mechanical neck pain: a case series. J Manipulative Physiol Ther 2007;30: 312-20.

34. Cleland JA, Fritz JM, Kulig K, Davenport TE, Eberhart S, Magel J, Childs J. Comparison of the effectiveness of three manual physical therapy techniques in a subgroup of patients with low back pain who satisfy a clinical prediction rule. Spine J 2009;34:2720-9.

35. Greene G. Red flags: essential factors in recognizing serious spinal pathology. Man Ther 2001;6:253-5.

36. Grieve GP. Common vertebral joint problems, 1st ed., 15. Edinburgh: Churcill Livingstone; 1981. p. 466.

37. Lee R. Kinematics of rotational mobilization of the lumbar spine. Clin Biomech 2001;16:481-8.

38. Pearcy MJ, Hindle RJ. Axial rotation of lumbar intervertebral joints in forward flexion. Proceeding of the Institution of Mechanical Engineers Part H. J Eng Med 1999;205:205-9.

39. Yang Z, Ting Ma H, Wang D, Lee R. Error analysis on spinal motion measurement using skin mounted sensors. Proceedings of the 30th Annual International Conference IEEE EMBS Engineering in Medicine and Biology Society, Vancouver; 2008. p. 4740-3.

40. Yang Z, Griffith J, Pope L, Sun L, Lee R. The accuracy of surface measurement for motion analysis of osteoporotic thoracolumbar spine. Proceedings of the 27th Annual International Conference IEEE EMBS Engineering in Med- icine and Biology Society, Shanghai; 2005. p. 6871-4.

41. Gibbons P, Tehan P. Patient positioning and spinal locking for lumbar spine rotation manipulation. Man Ther 2001;6:130-8.

42. Willet $\mathrm{E}$, Hebron $\mathrm{C}$, Krouwel $\mathrm{O}$. The initial effects of different rates of lumbar mobilizations on pressure pain thresholds in asymptomatic subjects. Man Ther 2010;15:173-8.

43. Krouwel $\mathrm{O}$, Hebron $\mathrm{C}$, Willet $\mathrm{E}$. An investigation into the potential hypoalgesic effects of different amplitudes of PA mobilizations on the lumbar spine as measured by pressure pain thresholds (PPT). Man Ther 2010;15:7-12. 